\title{
Chitosan nerve guidance channels with different geometries and their numerical analysis
}

\author{
Joana Gomes ${ }^{1}$, Jorge Belinha², Renato Natal ${ }^{3}$
}

${ }^{1}$ Institute of Science and Innovation in Mechanical and Industrial Engineering (INEGI), Faculty of Engineering, University of Porto, PT (joanamsgomes@gmail.com) ORCID 0000-0002-6250-340X, 2School of Engineering, Polytechnic of Porto, PT (job@isep.ipp.pt) ORCID 0000-0002-05397057, ${ }^{3}$ Faculty of Engineering, University of Porto, PT (rnatal@fe.up.pt) ORCID 0000-0002-7281-579X https://doi.org/10.24840/978-972-752-260-6_0010-0017

\begin{abstract}
Introduction: Peripheral nerve injuries are a major cause of permanent disabilities and have a negative impact on the quality of life of patients. The consequences of these injuries affect their daily living and work activities. Approximately $3 \%$ of trauma patients worldwide are affected by these injuries which are commonly attributed to direct mechanical trauma and to surgical resection. Although the knowledge about the pathophysiology of these injuries has been progressing, they still present as a challenge to surgeons. The most severe type of nerve injury is known as neurotmesis, which in the most extreme case results in a completely transected nerve. This originates a nerve gap, with total interruption of the structural integrity of the support structure of the nerve. There are different strategies that can be used to repair a peripheral nerve injury, being that both surgical and non-surgical approaches can be implemented. Whenever tensionless suture across the nerve gap is not possible, surgeons resort to the gold-standard technique which is the use of autologous nerve grafts (autografts). To overcome the disadvantages associated to this technique, nerve guidance channels (NGCs) made of biomaterials have been viewed as an alternative approach. One of the biomaterials that has been considered as a preferable candidate for peripheral nerve regeneration is chitosan. Methodology: In order to understand how these chitosan NGCs mechanically behave after being implanted at an injury site, discrete models of the NGCs containing a segment of a peripheral nerve were built using numerical methods to analyze them such as the finite element method (FEM) and the radial point interpolation method (RPIM). The discrete models had variable geometrical parameters: the length of the NGC and its wall thickness. The elastic constants considered were the Poisson's coefficient and the Young's modulus. Results and Discussion: Stress and displacement fields were obtained in order to comprehend the structural response of the NGCs when subjected to external forces. With the obtained results concerning stress and displacement distributions, it was possible to understand how the NGCs mechanically behave and which structural features are more indicated for their use. Conclusions: Although many advances have been made in the past decades, there is still the need to evolve and improve the different approaches to repair injuries in the peripheral nervous system. Numerical methods such as FEM and RPIM can numerically simulate the mechanical behavior of the chitosan NGCs and help to understand how they can be mechanically improved.
\end{abstract}

Keywords. Peripheral nerve, Chitosan, Finite element method, Meshless methods.

\section{INTRODUCTION}

\section{The repair of peripheral nerve injuries}

Peripheral nerve injuries affect $3 \%$ of trauma patients worldwide and they frequently lead to unsatisfactory functional recovery and life-long disabilities, which might prevent patients from resuming their jobs (Chiono \& Tonda-Turo, 2015; Faroni, Mobasseri, Kingham, \& Reid, 2015). Peripheral nerve injuries can occur after different traumatic events such as penetrating injury, crush, stretch, and ischemia (Ma, Fu, Jiang, \& Zhang, 2016). Other causes also include gunshot wounds and vehicle accidents, being the latter considered by some studies as the main etiological factor (Babaei-Ghazani, Eftekharsadat, Samadirad, Mamaghany, \& Abdollahian, 2017). It is known that younger patients as well as patients with more distal injuries fare better than older patients and more proximal injuries (Chhabra, Ahlawat, Belzberg, \& Andreseik, 2014). In cases where the injury occurs, for example, in a nerve of the hand the restoration of function can increase the independence and the well-being of the patient (Magown, Shettar, Zhang, \& Rafuse, 2015). The Seddon and Sunderland classifications are typically used to define the different types of injuries (Seddon, 1943; Sunderland, 1951). The most severe type of nerve injury is known as neurotmesis, which can result in the complete transection of the nerve originating a nerve gap. Consequently, the total interruption of the structural integrity of both the support structure of the nerve and its surrounding support structure occurs. This prevents a spontaneous recovery and leads to a complete motor, sensory and autonomic dysfunction 
(Chiono \& Tonda-Turo, 2015; Hainline, 2014; Houdek \& Shin, 2015). The repair of nerve injuries has been attempted for several years, being that the first successful nerve tubulation was performed in 1882. Since then, major advances have been made, both in the understanding of the nerve regeneration process and the strategies implemented to treat each type of injury (Adigüzel et al., 2016; Dalamagkas, Tsintou, \& Seifalian, 2016). After correct diagnosis of an injury, both surgical and non-surgical approaches can be implemented. The neurotmetic injuries require surgical intervention while other types of injuries can be treated by conservative methods (Lad, Nathan, Schubert, \& Boakye, 2010). The surgical intervention to treat a nerve gap can be an epineurial repair where both nerve ends are sutured together without tension (Grinsell \& Keating, 2014). Whenever tensionless suturing is not possible, surgeons resort to autografts which are considered the gold standard technique (Gu, Ding, Yang, \& Liu, 2011; Nectow, Marra, \& Kaplan, 2012). However, their use entails a series of drawbacks such as limited availability, morbidity at donor site, mismatch of donated nerves, loss of healthy nerve function and scar and neuroma formation (Ishikawa et al., 2009; Wu, Liu, Fang, Xiao, \& Wan, 2017). One of the alternatives is the use of NGCs made of biomaterials, either natural or synthetic (Chiono \& Tonda-Turo, 2015; Nectow et al., 2012; Zavan et al., 2008). Apart from the material, the architecture of the NGC can also differ. Some of the clinically available ones can have either a simple architecture or a more complex one, for they can be hollow tubes or they can have topographic cues (Grinsell \& Keating, 2014). Chitosan is a natural and hydrophilic copolymer of D-glucosamine and $\mathrm{N}$-acetyl-D-glucosamine units and it is obtained from full or partial $\mathrm{N}$ deacetylation of chitin, which is the second most abundant polysaccharide found in nature (Gu et al., 2011). It has a set of biological properties that makes it an ideal biomaterial for a variety of applications. It is biocompatible and biodegradable, it is easily fabricated and modified and it has antibacterial properties (Li, Xiao, Zhang, Zhao, \& Yang, 2017; Li et al., 2018).

\section{Numerical analysis}

FEM is an important technology in the modelling and simulation of advanced mechanical engineering systems. As a numerical method, it seeks an approximated solution of the distribution of field variables in the problem domain, which would be difficult to obtain analytically (Fish \& Belytschko, 2007; Liu \& Quek, 2003). This analysis is obtained by dividing the problem domain in several elements with simple geometry to which known physical laws are applied (Liu \& Quek, 2003). This numerical method can be used to create three-dimensional models of the peripheral nerve and the chitosan NGC to calculate tension values and deformations at specific points after applying loads (Behforootan, Chatzistergos, Naemi, \& Chockalingam, 2016; Piao, Yang, Li, \& Luo, 2015). Considering peripheral nerves, tissue mechanics and FEM analysis can be combined to explore their response when subjected to different stimuli (Giannessi, Stornelli, \& Sergi, 2017). As an alternative to the FEM, new discretization methods called meshless methods have been developed. They allow to deal with large distortions problems, occurring in soft materials, and they can be combined with scanning techniques, which is an advantage comparing with FEM (Belinha, 2016; Wah, 2008; Yagawa \& Yamada, 1996). Therefore, the goal of this work was to study the mechanical behavior of the chitosan NGCs with a segment of peripheral nerve inside of it. The models were analyzed considering both FEM and RPIM.

\section{METHODOLOGY}

The first step of this work was to construct the discrete models of the NGCs using the FEMAP software (student version). Two different geometric variables were considered: the length of 
the channel (LC) and the thickness of the wall (TW). In the first case, LC varied between 15 and $35 \mathrm{~mm}$ while TW was always $1 \mathrm{~mm}$. In the second case TW varied between 1 and $2.5 \mathrm{~mm}$ and LC was always $20 \mathrm{~mm}$. In both cases the initial $10 \mathrm{~mm}$ of the channel were hollow. In all cases, the section of the NGC that is not hollow is filled with a segment of a peripheral nerve, as illustrated in Figure 1. The numerical analysis with FEM and RPIM was performed using FEMAS software (cmech.webs.com). The mechanical properties for the elasto-static analyses considered were the Young's modulus and the Poisson's coefficient. These constants were obtained from studies in the literature regarding pure, hydrated chitosan samples. Under the premise that the NGCs are subjected to external forces after implantation, a displacement of 1 $\mathrm{mm}$ was applied to one node of one of the NGC extremity while the opposite one was fixed. As advanced discretization techniques, FEM and RPIM were used for the numerical analysis. Stress fields were obtained to comprehend the structural response of the NGCs when subjected to external forces.

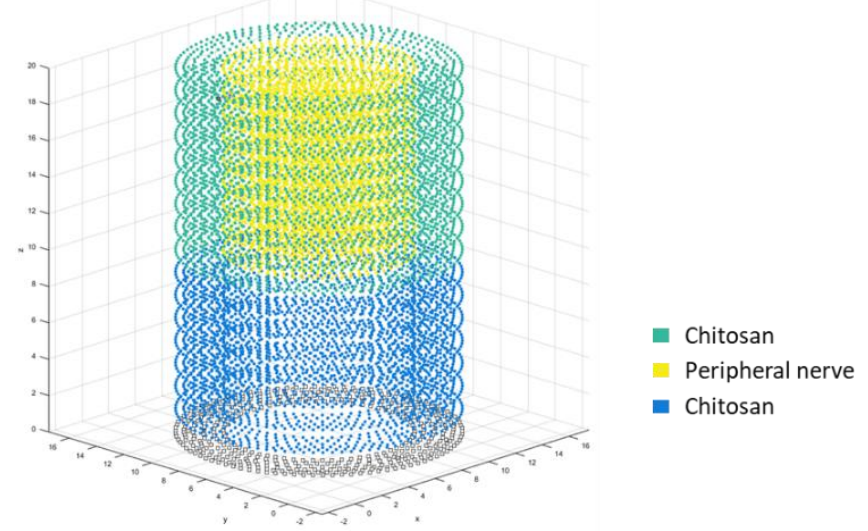

Figure 1. Representation of the discrete model of the chitosan NGC with the segment of nerve obtained from FEMAS software

\section{RESULTS}

From the numerical analysis of the chitosan NGCs with FEM and RPIM, it was possible to obtain stress and displacement fields concerning both geometric variables. In Figures 2 and 3 are represented the total displacement of the NGC when LC and TW vary, respectively. As for Figures 4 and 5, they represent the stress fields and the corresponding equivalent von Mises stresses when LC and TW vary, respectively. The displacement fields represented in Figure 2 show that the total displacement at the top of the channel, where the displacement was applied, the values are higher and similar for both values of LC. However, when comparing FEM with RPIM, the displacement values are lower in the latter. Considering Figure 3, the displacement values are higher when TW is smaller, which is observed in both numerical models. Figure 4 shows that the distribution of stresses is mainly concentrated in the lower part of the NGC, as well as in the opposite part of the channel (not seen). On the contrary, the lowest values of stress are concentrated in the section of the tube that contains the segment of nerve. In Figure 5, the behavior is similar as in Figure 4. In both cases, the values of stress are higher when LC and TW are $20 \mathrm{~mm}$ and $1.5 \mathrm{~mm}$, respectively. 


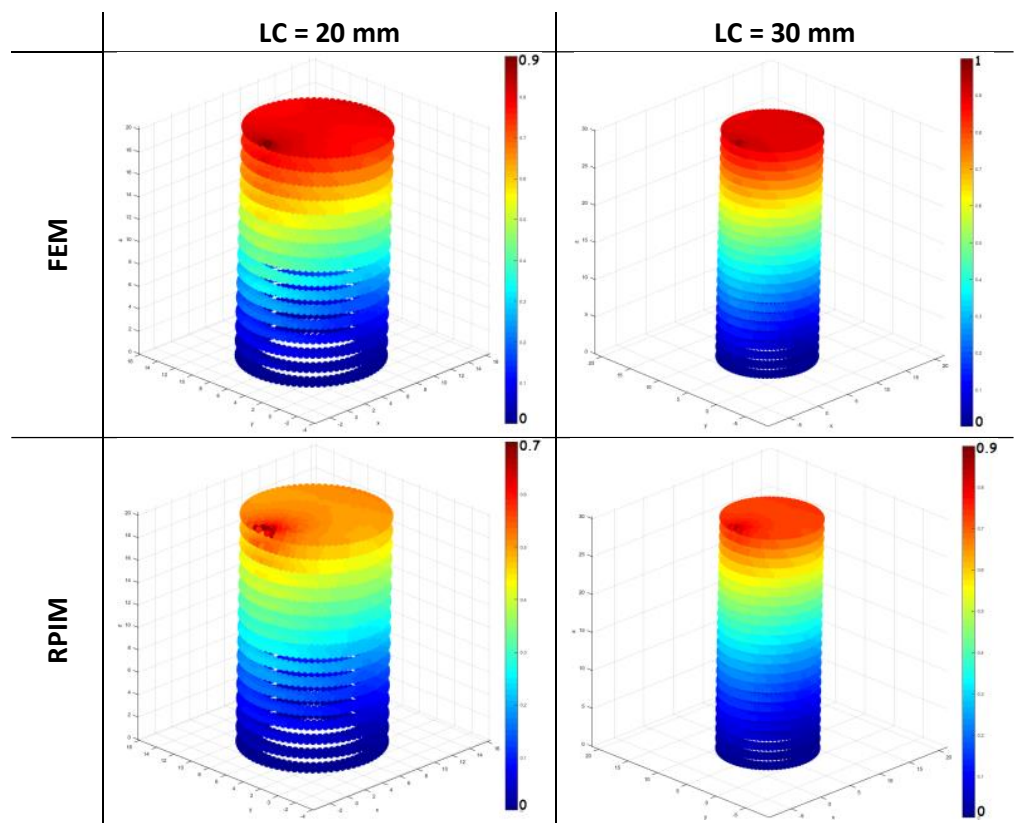

Figure 2. Representation of the total displacement in the model which geometric variable was the length of the channel

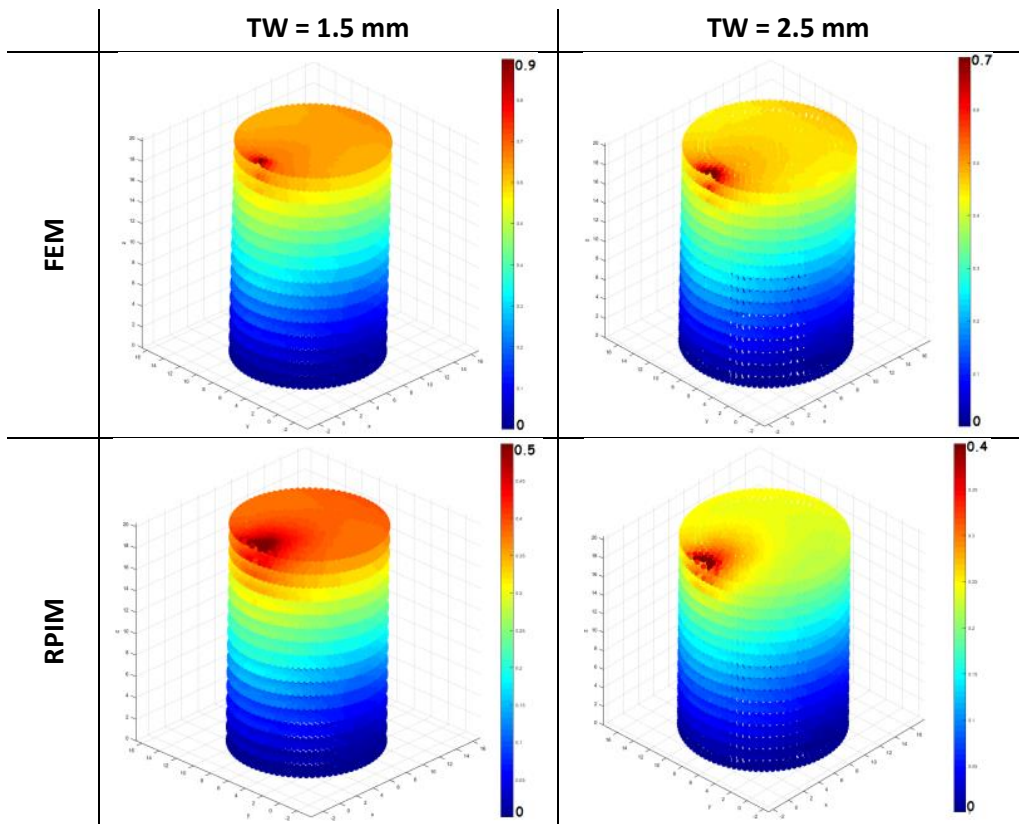

Figure 3. Representation of the total displacement in the model which geometric variable was the thickness of the wall 


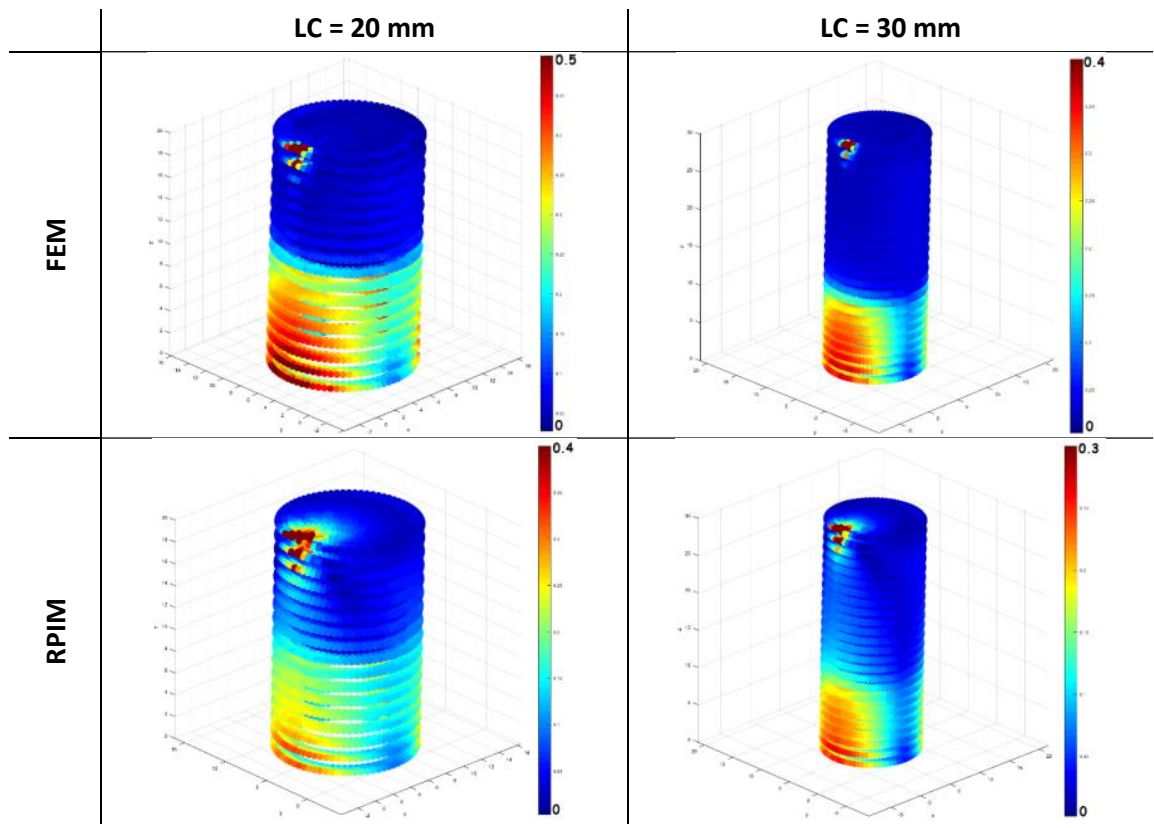

Figure 4. Distribution of the equivalent von Mises stresses in the model which geometric variable was the length of the channel

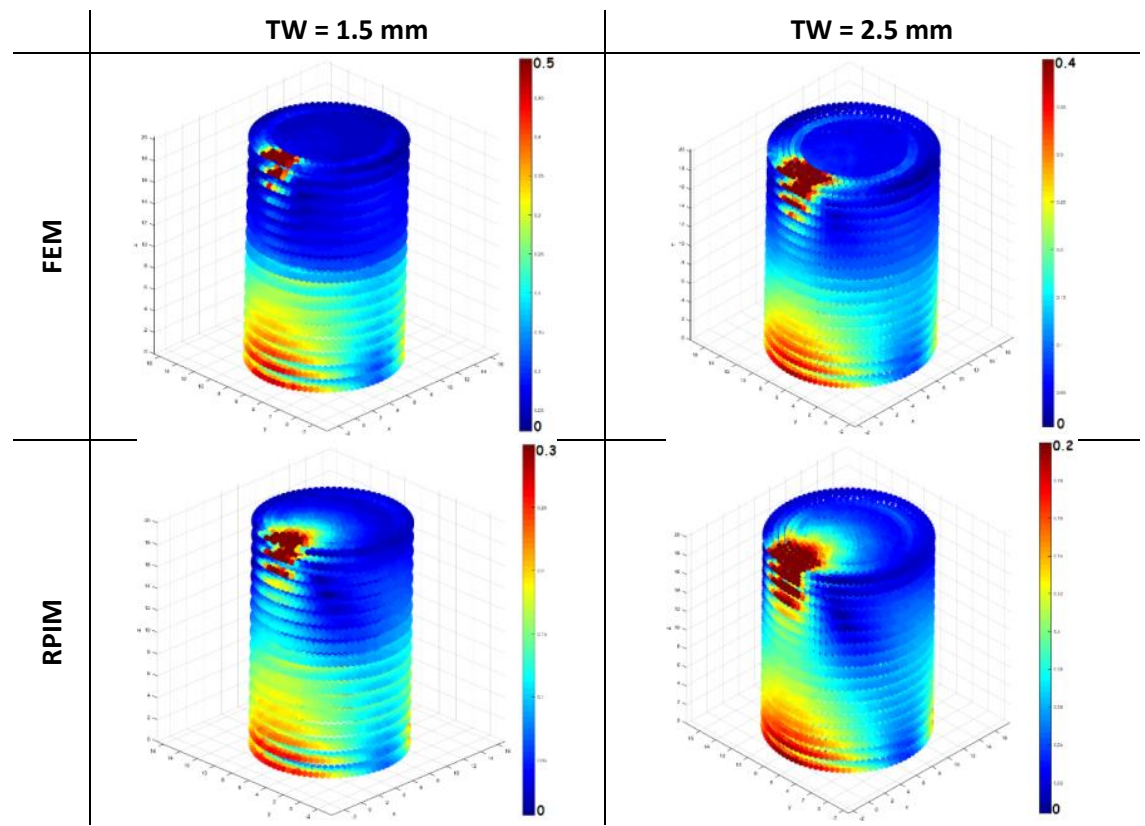

Figure 5. Distribution of the equivalent von Mises stresses in the model which geometric variable was the thickness of the wall

\section{DISCUSSION}

In this study, discrete models of a chitosan NGC were constructed in order to understand how they would behave when subjected to an external load. Stress and displacement fields were obtained, and the numerical analysis was performed using FEM and RPIM. As it was mentioned in the results section, the displacement values are higher when TW is smaller, which is true for FEM and RPIM. This could mean that NGCs with a thicker would be are more stable structures 
and less susceptible to movement. In some extent, this is a desired feature of the NGCs since they must maintain their physical structure while the nerve regeneration process occurs. Concerning the distribution of stresses, the higher concentration in the lower part of the NGC is due to stretching and compressing in opposite parts of the structure. This is related to the direction in which the displacement is applied. Concerning the numerical methods, there are visible differences in the range of values of displacement and stress. A refinement of the mesh of the discrete models might be necessary in order to decrease these differences between both methods.

\section{CONCLUSIONS}

Although many advances have been made in the past decades, there is still the need to evolve and improve the different approaches to repair injuries in the peripheral nervous system. Investigators have embarked in the mission of finding a strategy that allows surgeons to obtain good functional results in a consistent way. A promising one is the NGC made of chitosan used to bridge a nerve gap. With the help of numerical methods, such as FEM and RPIM, one can numerically simulate the mechanical behavior of NGCs made of chitosan and understand in what way they can be structurally improved. Ultimately this could lead to the development of a highly reliable and effective NGC and consequent repair of a peripheral nerve injury.

\section{Acknowledgments}

The authors truly acknowledge the funding provided by Ministério da Ciência, Tecnologia e Ensino Superior - Fundação para a Ciência e a Tecnologia (Portugal), under project funding MITEXPL/ISF/0084/2017. Additionally, the authors gratefully acknowledge the funding of Project NORTE-010145-FEDER-000022 - SciTech - Science and Technology for Competitive and Sustainable Industries, cofinanced by Programa Operacional Regional do Norte (NORTE2020), through Fundo Europeu de Desenvolvimento Regional (FEDER).

\section{References}

Adigüzel, E., Yaşar, E., Tecer, D., Güzelküçük, Ü., Taşkaynatan, M. A., Kesikburun, S., \& Özgül, A. (2016). Peripheral nerve injuries: Long term follow-up results of rehabilitation. Journal of Back and Musculoskeletal Rehabilitation, 1, 15. https://doi.org/10.3233/BMR-160681

Babaei-Ghazani, A., Eftekharsadat, B., Samadirad, B., Mamaghany, V., \& Abdollahian, S. (2017). Traumatic lower extremity and lumbosacral peripheral nerve injuries in adults: Electrodiagnostic studies and patients symptoms. Journal of Forensic and Legal Medicine, 52, 89-92. https://doi.org/10.1016/j.jflm.2017.08.010

Behforootan, S., Chatzistergos, P., Naemi, R., \& Chockalingam, N. (2016). Finite element modelling of the foot for clinical application: A systematic review. Medical Engineering \& Physics, 0, 1-11. https://doi.org/10.1016/j.medengphy.2016.10.011

Belinha, J. (2016). Meshless Methods in Biomechanics. Porto: Springer.

Chhabra, A., Ahlawat, S., Belzberg, A., \& Andreseik, G. (2014). Peripheral nerve injury grading simplified on MR neurography: As referenced to Seddon and Sunderland classifications. Indian Journal of Radiology and Imaging, 24(3), 217-224. https://doi.org/10.4103/0971-3026.137025

Chiono, V., \& Tonda-Turo, C. (2015). Trends in the design of nerve guidance channels in peripheral nerve tissue engineering. Progress in Neurobiology, 131, 87-104. https://doi.org/10.1016/j.pneurobio.2015.06.001

Dalamagkas, K., Tsintou, M., \& Seifalian, A. (2016). Advances in peripheral nervous system regenerative therapeutic strategies: A biomaterials approach. Materials Science and Engineering C, 65, 425-432. https://doi.org/10.1016/j.msec.2016.04.048 
Faroni, A., Mobasseri, S. A., Kingham, P. J., \& Reid, A. J. (2015). Peripheral nerve regeneration: Experimental strategies and future perspectives. Advanced Drug Delivery Reviews, 82, 160-167. https://doi.org/10.1016/j.addr.2014.11.010

Fish, J., \& Belytschko, T. (2007). A First Course in Finite Elements. The Journal of Chemical Physics (Vol. 17). John Wiley \& sons, Ltd. https://doi.org/10.1063/1.1747391

Galanakos, S., Zoubos, A., Mourouzis, I., Ignatiadis, I., Bot, A., \& Soucacos, P. (2013). Prognostic scoring system for peripheral nerve repair in the upper extremity. Microsurgery, 105-111. https://doi.org/10.1002/micr.22000

Giannessi, E., Stornelli, M. R., \& Sergi, P. N. (2017). A unified approach to model peripheral nerves across different animal species. PeerJ, 5, e4005. https://doi.org/10.7717/peerj.4005

Grinsell, D., \& Keating, C. P. (2014). Peripheral Nerve Reconstruction after Injury: A Review of Clinical and Experimental Therapies. BioMed Research International, 2014, 1-13. https://doi.org/10.1155/2014/698256

Gu, X., Ding, F., Yang, Y., \& Liu, J. (2011). Construction of tissue engineered nerve grafts and their application in peripheral nerve regeneration. Progress in Neurobiology, 93(2), 204-230. https://doi.org/10.1016/j.pneurobio.2010.11.002

Hainline, B. W. (2014). Peripheral Nerve Injury in Sports. CONTINUUM: Lifelong Learning in Neurology, 20(6, Sports Neurology), 1605-1628. Retrieved from http://journals.Iww.com/continuum/Fulltext/2014/12000/Peripheral_Nerve_Injury_in_Sports.12.aspx

Houdek, M. T., \& Shin, A. Y. (2015). Management and Complications of Traumatic Peripheral Nerve Injuries. Hand Clinics, 31(2), 151-163. https://doi.org/10.1016/j.hcl.2015.01.007

Ishikawa, N., Suzuki, Y., Dezawa, M., Kataoka, K., Ohta, M., Cho, H., \& Ide, C. (2009). Peripheral nerve regeneration by transplantation of BMSC-derived Schwann cells as chitosan gel sponge scaffolds. Journal of Biomedical Materials Research - Part A, 89(4), 1118-1124. https://doi.org/10.1002/jbm.a.32389

Lad, S. P., Nathan, J. K., Schubert, R. D., \& Boakye, M. (2010). Trends in median, ulnar, radial, and brachioplexus nerve injuries in the United States. Neurosurgery, 66(5), 953-960. https://doi.org/10.1227/01.NEU.0000368545.83463.91

Li, G., Xiao, Q., Zhang, L., Zhao, Y., \& Yang, Y. (2017). Nerve growth factor loaded heparin/chitosan scaffolds for accelerating peripheral nerve regeneration. Carbohydrate Polymers, 171, 39-49. https://doi.org/10.1016/j.carbpol.2017.05.006

Li, G., Xue, C., Wang, H., Yang, X., Zhao, Y., Zhang, L., \& Yang, Y. (2018). Spatially featured porous chitosan conduits with micropatterned inner wall and seamless sidewall for bridging peripheral nerve regeneration. Carbohydrate Polymers, 194(December 2017), 225-235. https://doi.org/10.1016/j.carbpol.2018.04.049

Liu, G. R., \& Quek, S. S. (2003). The finite element method - A practical course. Butterworth Heinemann.

Ma, M., Fu, Z., Jiang, B., \& Zhang, P. (2016). Electrophysiological and imaging outcomes analysis in patients with peripheral nerve injury treated with biodegradable conduit small-gap $(2 \mathrm{~mm})$ tubulization: A 5-year follow-up. International Journal of Clinical and Experimental Medicine, 9(2), 3770-3774.

Magown, P., Shettar, B., Zhang, Y., \& Rafuse, V. F. (2015). Direct optical activation of skeletal muscle fibres efficiently controls muscle contraction and attenuates denervation atrophy. Nature Communications, 6, 1-9. https://doi.org/10.1038/ncomms9506

Nectow, A. R., Marra, K. G., \& Kaplan, D. L. (2012). Biomaterials for the development of peripheral nerve guidance conduits. Tissue Engineering Part B: Reviews, 18(1), 40-50. https://doi.org/10.1089/ten.TEB.2011.0240

Piao, C.-D., Yang, K., Li, P., \& Luo, M. (2015). Autologous nerve graft repair of different degrees of sciatic nerve defect: stress and displacement at the anastomosis in a three-dimensional fnite element simulation model. Neural Regeneration Research, 10(5), 804-807. https://doi.org/10.4103/1673-5374.156986

Seddon, H. J. (1943). Three types of nerve injury. Brain, 66(4), 237-288.

Sunderland, S. (1951). A classification of peripheral nerve injuries producing loss of function. Brain, 74(4), 491-516.

Wah, B. (2008). Finite element method. Wiley Encyclopedia of Computer Science and Engineering, 1-12. Retrieved from http://onlinelibrary.wiley.com/doi/10.1002/9780470050118.ecse159/full 
Wu, H., Liu, J., Fang, Q., Xiao, B., \& Wan, Y. (2017). Establishment of nerve growth factor gradients on aligned chitosanpolylactide /alginate fibers for neural tissue engineering applications. Colloids and Surfaces B: Biointerfaces, 160, 598609. https://doi.org/10.1016/j.colsurfb.2017.10.017

Yagawa, G., \& Yamada, T. (1996). Free mesh method: A new meshless finite element method. Computational Mechanics, 18(5), 383-386. https://doi.org/10.1007/BF00376134

Zavan, B., Abatangelo, G., Mazzoleni, F., Bassetto, F., Cortivo, R., \& Vindigni, V. (2008). New 3D hyaluronan-based scaffold for in vitro reconstruction of the rat sciatic nerve. Neurological Research, 30(2), 190-196. https://doi.org/Doi $10.1179 / 174313208 \times 281082$ 